\title{
Management Impact of Imaging Brain Vesicular Monoamine Transporter Type 2 in Clinically Uncertain Parkinsonian Syndrome with ${ }^{18}$ F-AV133 and PET
}

\author{
Paschal K. Alexander ${ }^{1}$, Yenni Lie ${ }^{1}$, Gareth Jones ${ }^{1}$, Chomalaven Sivaratnam ${ }^{1}$, Svetlana Bozinvski ${ }^{1}$, Rachel S. Mulligan ${ }^{1}$, \\ Kenneth Young ${ }^{1}$, Victor L. Villemagne ${ }^{1-3}$, and Christopher C. Rowe ${ }^{1-3}$ \\ ${ }^{1}$ Department of Molecular Imaging and Therapy, Austin Health, Melbourne, Victoria, Australia; ${ }^{2}$ Florey Institute of Neuroscience and \\ Mental Health, University of Melbourne, Melbourne, Victoria, Australia; and ${ }^{3}$ Department of Medicine, University of Melbourne, \\ Melbourne, Victoria, Australia
}

\begin{abstract}
Idiopathic Parkinson disease is a common neurodegenerative disorder for which misdiagnosis occurs in up to $30 \%$ of patients after initial assessment and in 10\%-15\% even after long-term followup. Vesicular monoamine transporter type 2 (VMAT2) imaging with PET allows assessment of the integrity of the presynaptic dopaminergic pathway. We investigated the management impact of VMAT2 imaging in patients with clinically uncertain Parkinsonian syndromes. Methods: Forty-seven patients with clinically uncertain Parkinsonian syndromes (mean age \pm SD, $56.9 \pm 14.9$ y; age range, 21-80 y) were referred from movement disorder specialists. All participants underwent a 20-min PET acquisition $2 \mathrm{~h}$ after injection of $250 \mathrm{MBq}$ of ${ }^{18} \mathrm{~F}-\mathrm{AV}-133$, and the resulting images were quantitatively assessed. Clinical impact was recorded as high, moderate, or low based on diagnosis and management questionnaires completed by the referring specialists before and after release of the PET results. Management impact was high if there was a change in diagnostic category, moderate if there was a change in medication, and low if there was no change. Results: VMAT2 PET changed the diagnosis in $11(23 \%)$ and medication in $25(53 \%)$ participants. Management impact was high in $23 \%$, moderate in $38 \%$, and low in $39 \%$ of the participants. High diagnostic confidence increased from $11 \%$ of patients to $80 \%$ after the release of the scan results. Conclusion: ${ }^{18} \mathrm{~F}-\mathrm{AV}-133$ had substantial management impact in patients with clinically uncertain Parkinsonian syndromes. VMAT2 imaging with ${ }^{18} \mathrm{~F}-\mathrm{AV} 133$ might improve diagnosis, prognosis, and appropriate use of medication, translating into better patient outcomes.
\end{abstract}

Key Words: management impact; molecular imaging; Parkinson disease; PET; VMAT2

J Nucl Med 2017; 58:1815-1820

DOI: 10.2967/jnumed.116.189019

$\mathbf{I}$ diopathic Parkinson disease (iPD) is a common neurodegenerative disorder with an incidence of about 17 per 100,000 per year $(1,2)$. The pathophysiology of iPD includes loss of dopaminergic

Received Dec. 24, 2016; revision accepted Apr. 13, 2017.

For correspondence or reprints contact: Paschal K. Alexander, Department of Molecular Imaging and Therapy, Austin Health, 145 Studley Rd., Heidelberg, Victoria 3084, Australia.

E-mail: Paschal.Alexander@austin.org.au

Published online May 10, 2017.

COPYRIGHT (C) 2017 by the Society of Nuclear Medicine and Molecular Imaging. neurons in the substantia nigra and thereby loss of dopaminergic terminals in the striatum. This terminal loss correlates with the extrapyramidal symptoms of the disease. Although bradykinesia, rigidity, and resting tremor remain the clinical diagnostic criteria for iPD, reports of diagnostic accuracy compared with postmortem diagnosis varied from $70 \%$ in early stage disease to $90 \%$ in advanced stage in a tertiary referral movement disorder clinic (3-5). However, about 1 in 5 patients presenting with Parkinsonian features do not meet these diagnostic criteria (6) or may have additional clinical features suggesting another disease process. Under these circumstances, the patient may be labeled as clinically uncertain Parkinson syndrome (CUPS) (7). Several conditions might overlap with iPD, leading to misdiagnosis or diagnostic uncertainty. Among these, disorders in which the dopaminergic pathways are intact include essential tremor, dystonia, druginduced parkinsonism, and symptoms without dopaminergic deficit, or disorders in which there is dopaminergic neuronal loss plus more extensive neurodegeneration, such as multiplesystem atrophy, progressive supranuclear palsy, and corticobasal degeneration.

The shortcomings of clinical examination alone and the advantages of early diagnosis and early treatment necessitate a method for more accurate and early diagnosis.

The integrity of the nigrostriatal system can be evaluated noninvasively using PET and SPECT to provide clinical information that can assist in the early and differential diagnosis of iPD (8-14). Several radioligands have been developed for this purpose to image either presynaptic targets such as the dopamine transporter (DAT), vesicular monoamine transporter type 2 (VMAT2), dihydroxyphenylalanine decarboxylase activity, or postsynaptic D2 dopamine receptors (6). VMAT2 is involved in the uptake and storage of dopamine and other monoamines into presynaptic vesicles. It is mainly located at the nerve endings as well as nerve cell bodies and dendrites (15). Reduction in VMAT2 in the striatum reflects loss of nigrostriatal terminals $(16,17)$. The in vivo measurement of VMAT2 density has been shown to be potentially useful for the early and differential diagnosis of iPD $(18,19)$.

${ }^{18} \mathrm{~F}-\mathrm{AV}-133$ is an ${ }^{18} \mathrm{~F}$-labeled dihydrotetrabenazine analog. This compound binds selectively and with high affinity to VMAT2 $(19,20)$ and can sensitively detect monoaminergic terminal reductions in patients with Parkinson disease (PD) and dementia with Lewy bodies (15). Although DAT imaging has been shown to improve diagnostic accuracy for iPD and have substantial management impact in CUPS, VMAT2 imaging with ${ }^{18} \mathrm{~F}-\mathrm{AV}-133$ has 


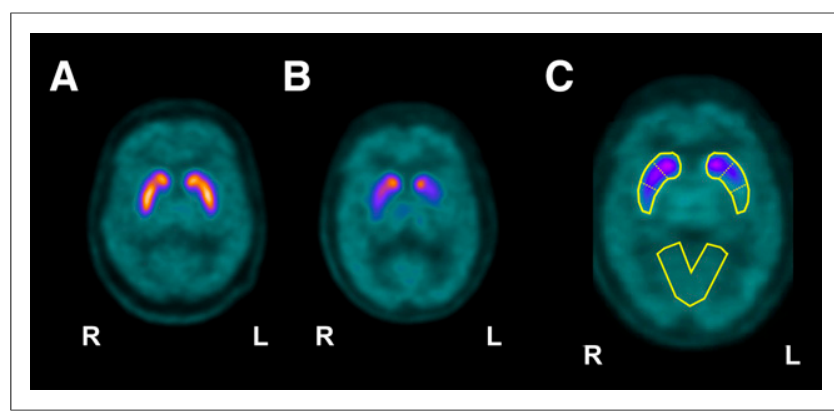

FIGURE 1. Representative ${ }^{18} \mathrm{~F}-\mathrm{AV}-133$ VMAT2 PET images of a normal age-matched volunteer (A) showing symmetric distribution of the tracer in basal ganglia, and PD patient (B) showing marked reduced and asymmetric tracer retention in basal ganglia, more pronounced in left putamen. Images were quantified using predefined region-of-interest template (C) that was applied on spatially normalized ${ }^{18} \mathrm{~F}-\mathrm{AV}-133$ images. Region-of-interest template sampled caudate nuclei, anterior and posterior putamen, and primary visual cortex that was used as reference region.

potential advantages such as better image quality and quantification, shorter time between tracer administration and scanning, shorter scan duration, and no requirement for prior blockade of the thyroid to prevent radioactive iodine uptake.

This study aimed to assess the impact of VMAT2 imaging using ${ }^{18} \mathrm{~F}-\mathrm{AV} 133$, on management of clinically uncertain Parkinson syndrome.

\section{MATERIALS AND METHODS}

\section{Study Design and Subject Selection}

The study was approved by the Austin Health Human Research Ethics Committee. All participants provided written informed consent before their participation in the study. The study used a prospective experimental study design. Study participants comprised patients with atypical features of parkinsonism who were referred from movement disorder specialists practicing in private or public clinics across Melbourne, Australia.

\section{Inclusion Criteria}

Participants were required to be older than $18 \mathrm{y}$ and English speaking. Participants were also required to have more than $7 \mathrm{y}$ of education and to have adequate visual and auditory acuity to complete the clinical and cognitive assessment.

\section{Exclusion Criteria}

Patients were excluded if they had a history of cancer (other than skin or in situ prostate cancer) within the previous $5 \mathrm{y}$. Persons were also excluded if they were unable to give informed written consent.

Pregnancy was excluded in women of childbearing age by blood test just before the scan.

\section{Assessments and Evaluation}

Each participant underwent neuropsychologic assessment and neurologic examination. The neuropsychologic assessment involved the MiniMental State Examination, clinical dementia rating, hospital anxiety and depression scale, logical memory score, and verbal fluency scores. The neurologic evaluation comprised the Hoehn and Yahr score and a motor subscale of the Unified Parkinson Disease Rating Scale score.

\section{Questionnaires}

The referring neurologists were required to complete diagnosis and management questionnaires at baseline and after the release of the AV-133 PET scan results.
The baseline questionnaire detailed the current management; the investigations completed; the most likely diagnosis from 6 categories (psychosomatic, dystonia, neurodegenerative, drug-induced, cerebrovascular disease, other [including essential tremor]); and the confidence, expressed as a percentage, in that diagnosis. If the clinician thought the diagnosis was a neurodegenerative disorder, they were required to specify whether this was thought to be PD, multiplesystem atrophy, progressive supranuclear palsy, cortical basal ganglionic degeneration, uncertain type, or other. Their confidence in the specific neurodegenerative disorder diagnosis was also recorded.

Details of proposed long-term management, including medication plan, referral to other health providers, and follow-up visit frequency, were also recorded. Subsequent questionnaires were issued after the scan results were released and the management impact determined on these same areas.

\section{Management Impact}

Outcome measures were defined as high, moderate, or low management impact. Management impact was defined as high if there was a change in diagnostic category from a progressive neurodegenerative disorder to a nondegenerative disorder or vice versa after the PET scan results, moderate if there was a resultant change in medication, or low if the results confirmed clinical diagnosis or the result was discrepant and ignored.

Confidence in diagnosis was rated into 3 categories as possible $(<50 \%)$, likely $(>50 \%)$, and highly likely/definite $(>90 \%)$ both before and after the release of scan results.

\section{PET Acquisition}

A 20-min emission PET scan was obtained $2 \mathrm{~h}$ after intravenous administration of approximately $250 \mathrm{MBq}$ of ${ }^{18} \mathrm{~F}-\mathrm{AV} 133$. The images were reconstructed using a 3-dimensional row-action maximumlikelihood algorithm (15) and were corrected for attenuation using a transmission scan from a rotating ${ }^{137} \mathrm{Cs}$ source.

\section{Image Analysis}

As previously reported, tissue ratios for the caudate nuclei and anterior and posterior putamen were calculated using the primary visual cortex as a reference region, and compared with a locally derived reference range (Fig. 1) (21). A reduction of more than 50\% in the most affected posterior putamen compared with the mean of a previously obtained normal control group (Fig. 2) was considered consistent with iPD or PD plus syndrome. This cutoff was based on observations from histopathologic studies that have documented loss of more than $50 \%$ of dopamine terminal markers in the posterior putamen in early symptomatic PD cases (22).

\section{RESULTS}

\section{Patient Demographics}

The study consisted of 47 participants. Patient demographics are displayed in Table 1 . There were 25 men (53\%) and 22 women (47\%) who ranged in age from 21 to $80 \mathrm{y}$. The mean age was 56.9 y. Only 1 participant had a Hoehn and Yahr score of zero, and no one had a score of 4.0 or 5.0. The mean baseline Mini-Mental State Examination score was 29 , and no participant had a score below 25 .

\section{Diagnosis Before Scanning}

Most patients (30 [64\%]) were initially diagnosed as having neurodegenerative disease, as seen in Table 2. Of these, 20 were thought to have iPD, 7 unknown, 1 multiple-system atrophy, 1 progressive supranuclear palsy, and 1 other. Seven (15\%) patients were thought to have a psychogenic condition; 4 (9\%) were 
diagnosed as dystonia and $3(6 \%)$ as drug-induced. Three patients were thought to have essential tremor (classified as other).

\section{Scan Findings}

Twenty-two patients (47\%) had abnormal scan findings (i.e., more than $50 \%$ reduction in ${ }^{18} \mathrm{~F}$-AV133 uptake in the posterior putamen). In these abnormal scan findings, reduction was more than $80 \%$ in $14,60 \%-79 \%$ in 5 , and $51 \%-59 \%$ in 2 (Fig. 2). Age correction was applied but had no impact on the measures.

\section{Diagnosis Change}

Data for the diagnosis change was obtained from the management impact questionnaires. More than $75 \%$ of the postscan release questionnaires were completed within 4 mo of the baseline questionnaire, and all were returned within a year.

The initial diagnosis was changed in $12(26 \%)$ patients after the release of the scan results. One of these was a change from dystonia to psychogenic and so was not considered as high impact based on our definition. Of the normal scan findings, the diagnosis was changed in $36 \%(9 / 25)$, and that value was $9 \%(2 / 22)$ for the abnormal scan findings.

\section{Medication Change}

With regard to medications, 53\% (25/47) of the participants had changes to their regimen after the scan results were released. When the scan results were abnormal, 54.5\% (12/22) had change, predominantly an increase in PD treatment, whereas $52 \%(13 / 25)$ of those with a normal scan finding had change, predominantly withdrawal of PD medications.

\section{Management Impact and Diagnostic Confidence}

Management impact was high in $23 \%$, moderate in $38 \%$, and low in $39 \%$ of patients (Fig. 3). Before the PET scan, clinician confidence was high (very likely/definite) for only $11 \%$ of the patients, but increased to $81 \%$ after the release of the scan results. Overall, there was an increase in diagnostic confidence in $74 \%$ of patients after the scan.

Confidence in the diagnosis increased after scanning, as illustrated in Table 3. This increase in confidence occurred whether the scan

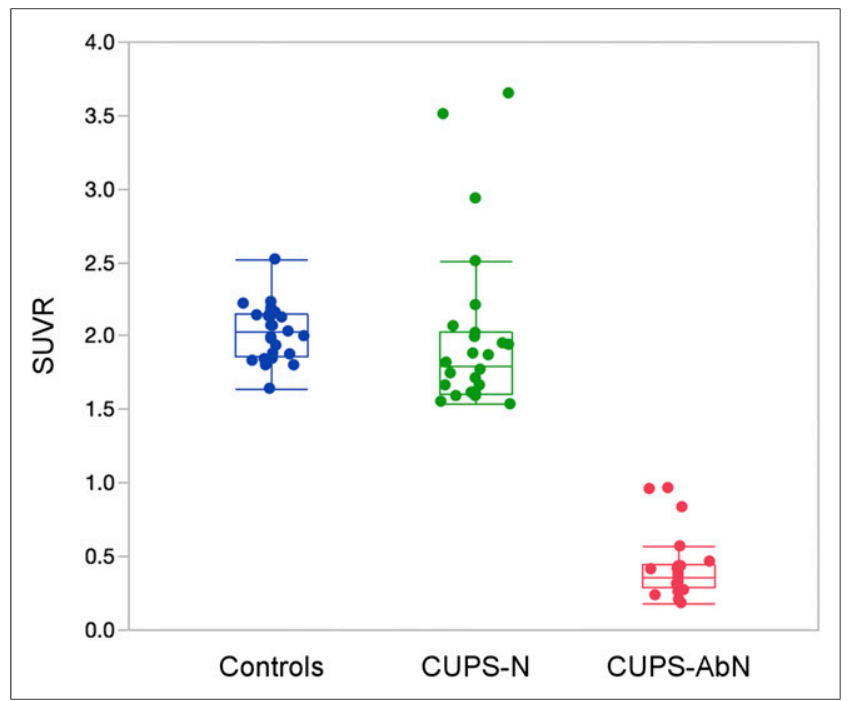

FIGURE 2. SUV ratios of posterior putamen in normal controls (blue), CUPS with normal scan findings (CUPS-N) (green), and CUPS with abnormal scan findings (CUPS-AbN) (red).
TABLE 1

Patient Demographics

\begin{tabular}{|c|c|}
\hline Characteristic & $n$ \\
\hline \multicolumn{2}{|l|}{ Age at baseline $(\mathrm{y})$} \\
\hline Mean \pm SD & $56.9 \pm 14.9$ \\
\hline Range (minimum-maximum) & $21-80$ \\
\hline \multicolumn{2}{|l|}{ Sex } \\
\hline Male & 25 \\
\hline Female & 22 \\
\hline Mean UPDRS score \pm SD for motor & $8.4 \pm 6$ \\
\hline UPDRS score \pm SD for rigidity & $0.7 \pm 1.0$ \\
\hline UPDRS score \pm SD for bradykinesia & $0.6 \pm 0.7$ \\
\hline \multicolumn{2}{|l|}{ Hoehn and Yahr score } \\
\hline 0 & 1 \\
\hline 1.0 , unilateral & 19 \\
\hline 1.5 , unilateral plus axial involvement & 4 \\
\hline $\begin{array}{l}\text { 2.0, bilateral disease without balance } \\
\text { impairment }\end{array}$ & 10 \\
\hline $\begin{array}{l}2.5 \text {, mild bilateral disease with recovery on } \\
\text { pull test }\end{array}$ & 5 \\
\hline 3.0, mild to moderate bilateral disease & 6 \\
\hline 4.0 , severe disability & 0 \\
\hline 5.0 , wheelchair-bound or bedridden & 0 \\
\hline \multicolumn{2}{|l|}{ MMSE score at baseline } \\
\hline Mean \pm SD & $29.1 \pm 1.4$ \\
\hline Range (minimum-maximum) & $25-30$ \\
\hline \multicolumn{2}{|c|}{$\begin{array}{l}\text { UPDRS }=\text { Unified Parkinson Disease Rating Scale; MMSE = } \\
\text { Mini-Mental State Examination. }\end{array}$} \\
\hline
\end{tabular}

results were abnormal $(16 / 22$ or $73 \%)$ or normal $(19 / 25$ or $76 \%)$. In addition, 4 of the 25 (16\%) normal scan findings resulted in a decrease in confidence with or without a change in diagnosis. This was only the case for 1 of the 22 abnormal scan results $(4.5 \%)$.

\section{DISCUSSION}

This study sought to determine whether the result of VMAT2 imaging with ${ }^{18} \mathrm{~F}-\mathrm{AV} 133$ affected the diagnosis and clinical management of patients with CUPS. Our findings indicated a moderate to high impact in $61 \%$ of the cases (38\% moderate and $23 \%$ high impact). We obtained an increase in diagnostic confidence in $74 \%$ of cases after the scan result was released and a $23 \%$ change in diagnosis.

These findings are comparable to studies using the SPECT DAT imaging agent ${ }^{123}$ I FP-CIT (DaTscan) when assessing loss of striatal dopaminergic innervation in CUPS. Seifert et al. reported that the DaTscan result led to a change of diagnosis in $31 \%$ and impacted clinical management in $58 \%$ of their patients (23). This is comparable to our values of $23 \%$ diagnosis change and $61 \%$ impact (combined moderate and high). However, their study was retrospective compared with our prospective design. Løkkegaard et al. demonstrated that the results of DaTscan led to a change of either diagnosis or clinical management in $27 \%$ of patients (24). 
TABLE 2

Scan Results and Diagnosis Before and After Results

\begin{tabular}{|c|c|c|c|}
\hline Patient no. & Scan result & Prescan diagnosis & Postscan diagnosis \\
\hline 1 & Positive & Dystonia & Neurodegenerative \\
\hline 2 & Positive & Neurodegenerative & Neurodegenerative \\
\hline 3 & Negative & Dystonia & Psychogenic \\
\hline 4 & Positive & Neurodegenerative & Neurodegenerative \\
\hline 5 & Positive & Neurodegenerative & Neurodegenerative \\
\hline 6 & Negative & Neurodegenerative & Other-benign tremor \\
\hline 7 & Positive & Neurodegenerative & Neurodegenerative \\
\hline 8 & Positive & Neurodegenerative & Neurodegenerative \\
\hline 9 & Positive & Neurodegenerative & Neurodegenerative \\
\hline 10 & Positive & Neurodegenerative & Neurodegenerative \\
\hline 11 & Negative & Drug-induced & Drug-induced \\
\hline 12 & Negative & Psychogenic & Psychogenic \\
\hline 13 & Negative & Neurodegenerative & Other \\
\hline 14 & Negative & Neurodegenerative & Other-benign tremor \\
\hline 15 & Positive & Neurodegenerative & Neurodegenerative \\
\hline 16 & Positive & Neurodegenerative & Neurodegenerative \\
\hline 17 & Negative & Psychogenic & Psychogenic \\
\hline 18 & Negative & Neurodegenerative & Psychogenic \\
\hline 19 & Negative & Psychogenic & Psychogenic \\
\hline 20 & Positive & Neurodegenerative & Neurodegenerative \\
\hline 21 & Positive & Neurodegenerative & Neurodegenerative \\
\hline 22 & Positive & Neurodegenerative & Neurodegenerative \\
\hline 23 & Negative & Psychogenic & Psychogenic \\
\hline 24 & Positive & Neurodegenerative & Neurodegenerative \\
\hline 25 & Negative & Other & Other \\
\hline 26 & Positive & Neurodegenerative & Neurodegenerative \\
\hline 27 & Positive & Neurodegenerative & Neurodegenerative \\
\hline 28 & Negative & Neurodegenerative & Neurodegenerative \\
\hline 29 & Negative & Other & Other \\
\hline 30 & Negative & Psychogenic & Psychogenic \\
\hline 31 & Positive & Neurodegenerative & Neurodegenerative \\
\hline 32 & Negative & Neurodegenerative & Psychogenic \\
\hline 33 & Positive & Neurodegenerative & Neurodegenerative \\
\hline 34 & Positive & Other & Neurodegenerative \\
\hline 35 & Negative & Psychogenic & Psychogenic \\
\hline 36 & Negative & Neurodegenerative & Dystonia \\
\hline 37 & Negative & Neurodegenerative & Cerebral vascular disease \\
\hline 38 & Negative & Drug-induced & Drug-induced \\
\hline 39 & Negative & Dystonia & Dystonia \\
\hline 40 & Positive & Neurodegenerative & Neurodegenerative \\
\hline 41 & Positive & Neurodegenerative & Neurodegenerative \\
\hline 42 & Negative & Neurodegenerative & Drug-induced \\
\hline 43 & Negative & Neurodegenerative & Other \\
\hline 44 & Positive & Neurodegenerative & Neurodegenerative \\
\hline 45 & Negative & Psychogenic & Psychogenic \\
\hline 46 & Negative & Drug-induced & Drug-induced \\
\hline 47 & Negative & Dystonia & Dystonia \\
\hline
\end{tabular}

1818 The Journal of Nuclear Medicine • Vol. 58 • No. 11 - November 2017 


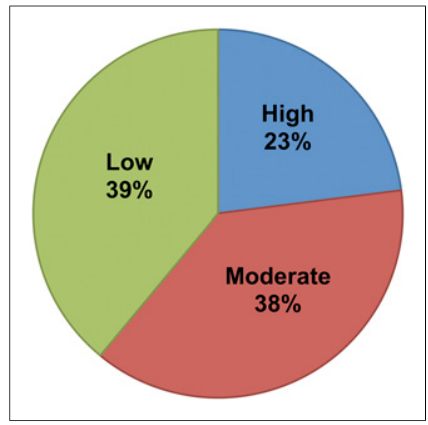

FIGURE 3. Postscan management impact.

Clinician confidence was also affected by the ${ }^{18} \mathrm{~F}-\mathrm{AV} 133$ scans. Clinician confidence increased in $74 \%$ of the cases after the scan result was revealed. DaTscan studies conducted by both Kupsch et al. (25) and Seifert et al. (23) also demonstrated significant changes in confidence in diagnosis after release of results. Interestingly, Kupsch et al. followed up after $4 \mathrm{wk}$, $12 \mathrm{wk}$, and $1 \mathrm{y}$ and reported a further increase in confidence in the diagnosis at each follow-up interval (25), indicating that diagnosis assisted by striatal dopaminergic innervation imaging remained robust over time.

In our study, clinician confidence was equally increased whether the scan was normal or abnormal. This contrasts with the findings by Catafau et al. (7) in their study with DaTscan. They found an increase in confidence when the scan results were abnormal but a decrease in confidence when the scan results were normal (7).

The medication regimen was affected in $53 \%$ of the patients. The changes ranged from commencement of a new agent and cessation of an agent to change in the dose. Studies conducted with DaTscan also attributed a significant portion of their management change after scanning to medication changes (7,23-25).

Of the 22 patients with abnormal scan findings, 14 had a reduction in VMAT2 binding compared with the mean of controls, of more than $80 \%$. This highlights the fact that diagnostic uncertainty can manifest even with a high degree of dopaminergic loss.

We chose a reduction in relative binding in the posterior putamen of greater than $50 \%$ to be abnormal. This value was chosen because previous postmortem studies have shown that a reduction of about $50 \%$ of dopaminergic neurons was necessary to produce clinical symptoms sufficient for the diagnosis of PD to be made (22). In our group, $50 \%$ reduction corresponds to 3 SDs below the mean of a reference group consisting of healthy normal adults who underwent VMAT2 PET imaging with ${ }^{18} \mathrm{~F}-\mathrm{AV} 133$. As such, we believe that we are justified in using that value for this application though it would not be appropriate if preclinical detection of iPD is the goal.

There were some limitations to this study. First, there was no pathologic confirmation of the diagnosis in those with abnormal scans nor was there confirmation from longitudinal clinical followup. Prior studies have demonstrated a high concordance of DaTscan findings with postmortem diagnosis, and similar studies would be useful for VMAT2 imaging.

The study population was generally referred from movement disorder specialists in Melbourne, and so the demographics of the

TABLE 3

Clinical Confidence Before and After Scanning

\begin{tabular}{lcc}
\hline \multicolumn{1}{c}{ Category } & $\begin{array}{c}\text { Number before } \\
\text { scanning }\end{array}$ & $\begin{array}{c}\text { Number after } \\
\text { scanning }\end{array}$ \\
\hline Possible & 15 & 6 \\
Likely & 27 & 3 \\
Very likely/definite & 5 & 38 \\
\hline
\end{tabular}

study participants reflect that of the local, predominantly Caucasian, population. There was no control arm in this study such as CUPS patients without VMAT2 imaging. Therefore, it is possible that diagnosis and clinician confidence changed because of the passage of time or repeated clinical assessment. A controlled study is needed to address this issue.

The findings in this study provide further evidence for the use of imaging as a complementary aide in managing CUPS. Although the findings are similar to those obtained with the SPECT imaging agent DaTscan, ${ }^{18} \mathrm{~F}-\mathrm{AV} 133$ PET has logistic advantages including shorter uptake and scanning times and no patient preparation. ${ }^{18} \mathrm{~F}$ AV133 PET also produces high-quality images that allow more accurate quantification, and this may be important if monitoring change over time as in therapeutic drug trials.

\section{CONCLUSION}

Significant impact in management and confidence in the diagnosis was derived from imaging VMAT2 with ${ }^{18} \mathrm{~F}-\mathrm{AV} 133$ and PET in patients with CUPS.

\section{DISCLOSURE}

This was an investigator-initiated study supported by a grant from Avid Radiopharmaceuticals. This study was also supported in part by funds from the Austin Hospital Medical Research Foundation. Christopher Rowe, MD, has received research grants from GE Healthcare, Piramal Imaging, Navidea, and Avid Radiopharmaceuticals. Victor L. Villemagne, MD, has been an honorary speaker for Avid Radiopharmaceuticals. No other potential conflict of interest relevant to this article was reported.

\section{ACKNOWLEDGMENTS}

We thank Drs. Andrew Evans, David Williams, John Drago, Andrew Hughes, John Merory, Katrina Reardon, and Katya Kotschet for their role in patient recruitment.

\section{REFERENCES}

1. Kasten M, Chade A, Tanner CM. Epidemiology of Parkinson's disease. Handb Clin Neurol. 2007;83:129-151.

2. Twelves D, Perkins KS, Counsell C. Systematic review of incidence studies of Parkinson's disease. Mov Disord. 2003;18:19-31.

3. Hughes AJ, Daniel SE, Lees AJ. Improved accuracy of clinical diagnosis of Lewy body Parkinson's disease. Neurology. 2001;57:1497-1499.

4. Rajput AH, Rozdilsky B, Rajput A. Accuracy of clinical diagnosis in Parkinsonism: a prospective study. Can J Neurol Sci. 1991;18:275-278.

5. Hughes AJ, Daniel SE, Kilford L, Lees AJ. Accuracy of clinical diagnosis of idiopathic Parkinson's disease: a clinico-pathological study of 100 cases. $J$ Neurol Neurosurg Psychiatry. 1992;55:181-184.

6. Cummings JL, Henchcliffe C, Schaier S, Simuni T, Waxman A, Kemp P. The role of dopaminergic imaging in patients with symptoms of dopaminergic system neurodegeneration. Brain. 2011;134:3146-3166.

7. Catafau AM, Tolosa E. DaTSCAN Clinically Uncertain Parkinsonian Syndromes Study Group. Impact of dopamine transporter SPECT using ${ }^{123}$ I-ioflupane on diagnosis and management of patients with clinically uncertain Parkinsonian syndromes. Mov Disord. 2004;19:1175-1182.

8. Jennings DL, Seibyl JP, Oakes D, Eberly S, Murphy J, Marek K. ( ${ }^{123}$ I) beta-CIT and single-photon emission computed tomographic imaging vs clinical evaluation in Parkinsonian syndrome: unmasking an early diagnosis. Arch Neurol. 2004;61:1224-1229.

9. Ravina B, Eidelberg D, Ahlskog JE, et al. The role of radiotracer imaging in Parkinson disease. Neurology. 2005;64:208-215.

10. Wong DF, Yung B, Dannals RF, et al. In vivo imaging of baboon and human dopamine transporters by positron emission tomography using $\left[{ }^{11} \mathrm{C}\right]$ WIN 35,428. Synapse. 1993;15:130-142. 
11. Villemagne V, Yuan J, Wong DF, et al. Brain dopamine neurotoxicity in baboons treated with doses of methamphetamine comparable to those recreationally abused by humans: evidence from $\left[{ }^{11} \mathrm{C}\right]$ WIN-35,428 positron emission tomography studies and direct in vitro determinations. J Neurosci. 1998;18: 419-427.

12. Pirker W, Djamshidian S, Asenbaum S, et al. Progression of dopaminergic degeneration in Parkinson's disease and atypical Parkinsonism: a longitudinal b-CIT SPECT study. Mov Disord. 2002;17:45-53.

13. Frey KA, Koeppe RA, Kilbourn MR, et al. Presynaptic monoaminergic vesicles in Parkinson's disease and normal aging. Ann Neurol. 1996;40:873-884.

14. Stoessl AJ. Positron emission tomography in premotor Parkinson's disease. Parkinsonism Relat Disord. 2007;13(suppl 3):S421-S424.

15. Okamura N, Villemagne VL, Drago J, et al. In vivo measurement of vesicular monoamine transporter type 2 density in Parkinson disease with ${ }^{18} \mathrm{~F}-\mathrm{AV}-133$. J Nucl Med. 2010;51:223-228.

16. Nirenberg MJ, Chan J, Liu Y, Edwards RH, Pickel VM. Ultrastructural localization of the vesicular monoamine transporter-2 in midbrain dopaminergic neurons: potential sites for somatodendritic storage and release of dopamine. J Neurosci. 1996;16:4135-4145.

17. Martin WR, Wieler M, Stoessl AJ, Schulzer M. Dihydrotetrabenazine positron emission tomography imaging in early, untreated Parkinson's disease. Ann Neurol. 2008;63:388-394

18. Chen MK, Kuwabara H, Zhou Y, et al. VMAT2 and dopamine neuron loss in a primate model of Parkinson's disease. J Neurochem. 2008;105:78-90.
19. Kung MP, Hou C, Goswami R, Ponde DE, Kilbourn MR, Kung HF. Characterization of optically resolved 9-fluoropropyl-dihydrotetrabenazine as a potential PET imaging agent targeting vesicular monoamine transporters. Nucl Med Biol. 2007;34:239-246.

20. Goswami R, Ponde DE, Kung MP, Hou C, Kilbourn MR, Kung HF. Fluoroalkyl derivatives of dihydrotetrabenazine as positron emission tomography imaging agents targeting vesicular monoamine transporters. Nucl Med Biol. 2006;33: 685-694.

21. Villemagne VL, Okamura N, Pejoska S, et al. Differential diagnosis in Alzheimer's disease and dementia with Lewy bodies via VMAT2 and amyloid imaging. Neurodegener Dis. 2012;10:161-165.

22. Kordower JH, Olanow CW, Dodiya HB, et al. Disease duration and the integrity of the nigrostriatal system in Parkinson's disease. Brain. 2013;136(pt 8):24192431.

23. Seifert KD, Weiner JI. The impact of DaTscan on the diagnosis and management of movement disorders: a retrospective study. Am J Neurodegener Dis. 2013; 2:29-34.

24. Løkkegaard A, Werdelin LM, Friberg L. Clinical impact of diagnostic SPET investigations with a dopamine re-uptake ligand. Eur J Nucl Med Mol Imaging. 2002;29:1623-1629.

25. Kupsch AR, Bajaj N, Weiland F, et al. Impact of DaTscan SPECT imaging on clinical management, diagnosis, confidence of diagnosis, quality of life, health resource use and safety in patients with clinically uncertain Parkinsonian syndromes: a prospective 1-year follow-up of an open-label controlled study. J Neurol Neurosurg Psychiatry. 2012;83:620-628. 